\title{
Supercapacitors on demand: all-printed energy storage devices with adaptable design
}

Robert Brooke, Jesper Edberg, Mehmet Girayhan Say, Anurak Sawatdee, Andrea Grimoldi, Jessica Ahlin, Göran Gustafsson, Magnus Berggren and Isak Engquist

The self-archived postprint version of this journal article is available at Linköping University Institutional Repository (DiVA):

http://urn.kb.se/resolve?urn=urn:nbn:se:liu:diva-164472

N.B.: When citing this work, cite the original publication.

Brooke, R., Edberg, J., Say, M. G., Sawatdee, A., Grimoldi, A., Ahlin, J., Gustafsson, G., Berggren, M., Engquist, I., (2019), Supercapacitors on demand: all-printed energy storage devices with adaptable design, FLEXIBLE AND PRINTED ELECTRONICS, 4(1), 015006. https://doi.org/10.1088/2058$8585 /$ aafc $4 \mathrm{f}$

Original publication available at:

https://doi.org/10.1088/2058-8585/aafc4f

Copyright: IOP Publishing (Hybrid Open Access)

http://www.iop.org/ 
Supercapacitors on Demand: All-Printed Energy Storage Devices with

\section{Adaptable Design}

Robert Brooke $^{\mathrm{a}}$, Jesper Edberg ${ }^{\mathrm{a} *}$, Mehmet Girayhan Say ${ }^{\mathrm{b}}$, Anurak Sawatdee $^{\mathrm{a}}$, Andrea Grimoldi ${ }^{\mathrm{b}}$, Jessica Åhlinª, Göran Gustafsson ${ }^{\mathrm{a}}$, Magnus Berggren ${ }^{\mathrm{b}}$, Isak Engquist ${ }^{\mathrm{b}}$

*Corresponding author: jesper.edberg@,ri.se

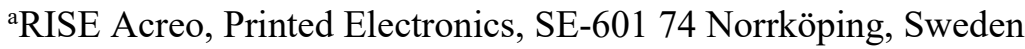

bLaboratory of Organic Electronics, Department of Science and Technology, Linköping University, SE-601 74 Norrköping, Sweden

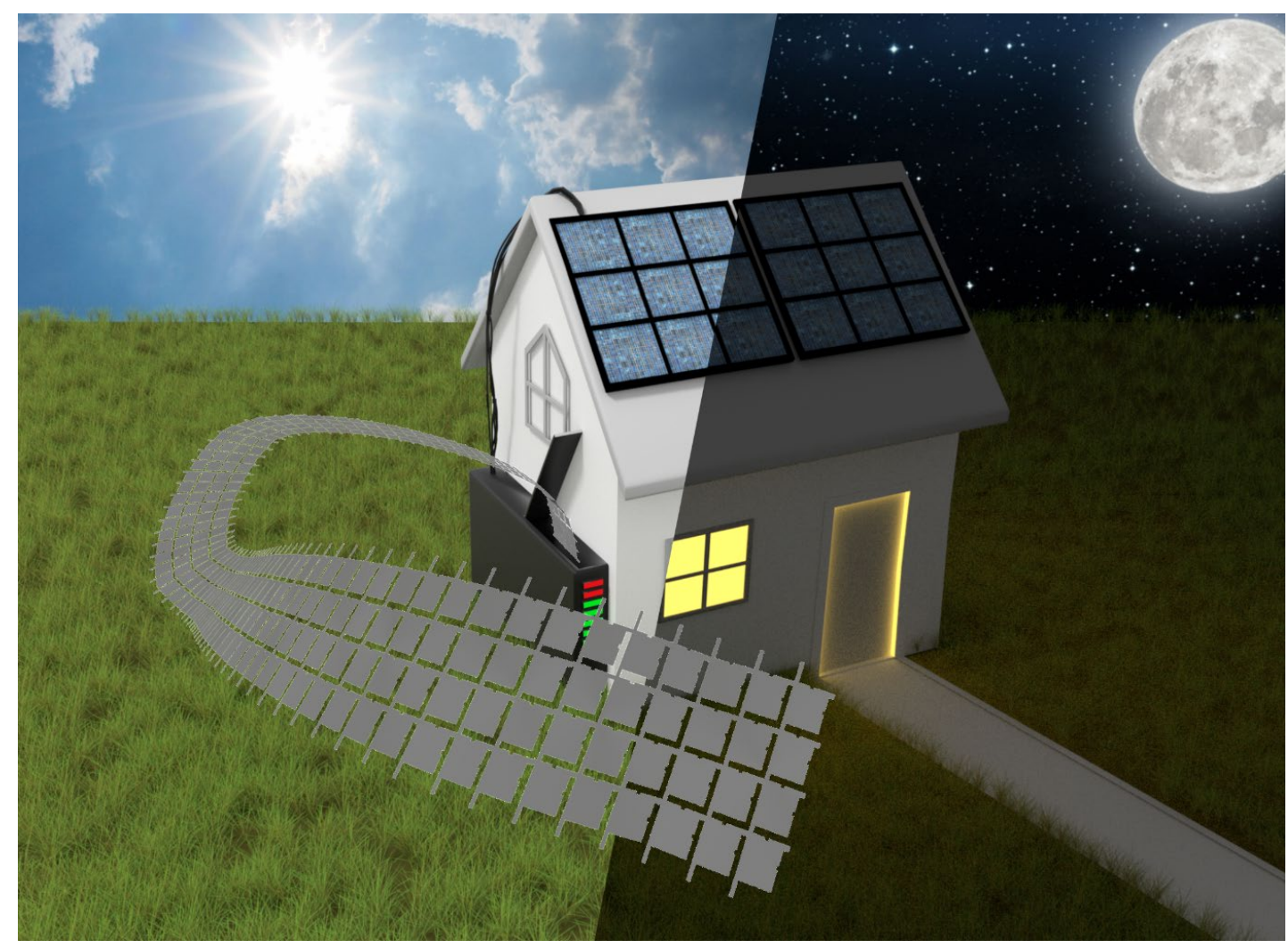

Cover/TOC image 
Abstract

Demands in the storage of energy have increased for many reasons, in part driven by household photovoltaics, electric grid balancing, along with portable and wearable electronics. These are fast-growing and differentiated applications that urge for large volume and/or highly distributed electrical energy storage, which then requires environmentally friendly, scalable and flexible materials and manufacturing techniques. However, the limitations on current inorganic technologies have driven research efforts to explore organic and carbon-based alternatives. Here, we report a conducting polymer:cellulose composite that serves as the active material in supercapacitors which has been incorporated into all printed energy storage devices. These devices exhibit a specific capacitance of $\approx 90 \mathrm{~F} / \mathrm{g}$ and an excellent cyclability $(>10,000$ cycles $)$. Further, a design concept coined 'supercapacitors on demand' is presented, which is based on a printing-cutting-folding procedure, that provide us with a flexible production protocol to manufacture supercapacitors with adaptable configuration and electrical characteristics.

\section{Introduction}

The energy demands in our society are ever increasing due to the digital age, growing populations and general improved standards of living. At the same time, a shift from fossil fuels to renewable energy sources, such as wind and solar power, will require intermediate energy storage solutions to balance the needs in society with availability in the power grids. Current energy storage devices such as batteries and supercapacitors typically rely on inorganic materials that cannot meet society's future energy needs due to limited material resources, manufacturing costs, environmental aspects and infrastructure. (1) Energy storage requirements are also growing beyond the industrial scene with small, light and flexible energy storage technologies needed for sensors and other components in portable and wearable electronics. (2-4) Therefore, society needs sustainable energy storage solutions that can be employed to store large amounts of energy for industrial requirements but can also be adapted for smaller, and portable applications. Printed electronics is one avenue of producing energy storage devices via potentially high-throughput procedures at the same time achieving customizable designs at low costs. 
Screen and stencil printing are both versatile yet simple printing techniques that can be used to coat a variety of shape, size and material surfaces such as glass, clothes or plastics. (9) The templates used in screen and stencil printing are screen meshes or open masks, respectively, designed to print over large areas with resolutions down to tens of microns. A metal blade (stencil printing) or a rubber squeegee (screen printing) forces and transfers the ink (possessing appropriate viscosity, wetting, etc properties) through the mesh or mask pattern onto the substrate underneath. (10) Registration or alignment markings allows multiple and sequential layers of printing possible, with a high level of accuracy that is crucial in producing printed electronic components or devices. Various levels of automation are possible by using advanced printing machines, such as in-line registration, up/downloading, ink curing and more.

Other advantages of screen and stencil printing include the elimination of soldering, welding and other connection techniques for traditional energy storage solutions which are often mechanically weak when flexed or bent. In the past, researchers have taken advantage of the unique features of printed electronics for energy storage technologies. Kumar et al. (11) presented an all screen printed and rechargeable $\mathrm{Zn}-\mathrm{Ag}_{2} \mathrm{O}$ battery, which due to the absence of interconnections possessed impressive stretchability up to $100 \%$ under operation.

Stencil printing energy storage solutions have also been reported previously by Gaikwad et al. (12) constructing a $14-\mathrm{V}$ flexible $\mathrm{Zn}-\mathrm{MnO}_{2}$ battery. Here, the layers were deposited one after the other via stencil printing, avoiding the need for assembly processes.

The use of organic electronic materials as the active electrode in batteries and supercapacitors, hold promise for several key-features of relevance for the growing need of electrical energy storage, such as $(i)$ replacing expensive and rare inorganic materials, (ii) avoiding the limitation of material abundance, (iii) providing low costs, and (iv) potentially environmentally friendly energy solutions. (1, 13) Within the field of organic electronics, two classes of materials hold the most promise for energy storage applications by providing sufficient conductivity and capacity; carbon based-materials(14-16) and conductive polymers(17-19). The focus of the researcher presented herein is on conductive polymers. Conducting polymers represent an "organic" alternative in electrical energy storage and is 
gaining increased attention within the field of printed electronics, in general, and energy storage, in particular, due to their excellent electrical properties and stability in air. Poly(3,4ethylenedioxythiophene):poly(styrene sulfonate) (PEDOT:PSS) is the most studied and explored conducting polymer system and has been employed in various printed electronics applications, such as the electrode and conductor in printed display devices $(20,21)$ and in supercapacitors $(22,23)$.

Previous reports highlighting the ability of conducting polymers to compete with their inorganic counterparts have shown encouraging results. (24) Screen printed polypyrrole/silver nanocomposites were recently incorporated into energy storage micro-devices. The authors reported impressive results with acceptable degradation over 10,000 cycles in addition to good performance while flexed. The devices were designed in a lateral configuration which in a practical setting may be a disadvantage although it does eliminate a lamination step.

Recently, we reported the power paper concept, which is a material system based on a composite of cellulose nanofibrils (CNF) and PEDOT:PSS. $(18,19,25,26)$ This materials system allows for a mechanically robust, nano-porous and scalable electroactive network (properties provided by the CNF component) and swift electronic conduction and ion exchange (provided by the conducting polymer). The power paper has been incorporated as the electrode in symmetric pseudocapacitors but has yet to be shown in large scale manufacturing. (19) In this report, we present the development of the stencil printable power paper ink and report its performance while incorporating this material in supercapacitors manufactured using a flexible "on demand" production protocol. The resulting technology is an energy storage solution that is customizable and possible to tailor-make for specific applications and specifications. Disconnections can be introduced within the design to configure supercapacitors connected in series or in parallel, thus enabling us to increase or reduce the operational voltage depending on the targeted specification. First, we report the investigation of the fundamental material properties of the power paper ink. Then, we describe and present the printing procedures and the electrical characterization of the resulting supercapacitors. Finally, we showcase some of the possibilities of the supercapacitor-on-demand concept. This type of printed and customizable design of 
electric energy storage, based on organic electroactive materials, provides one route towards supplying the demand for scalable energy solutions in our society.

\section{Experimental}

\section{Materials}

Orgacon DRY poly(3,4-ethylenedioxythiophene):poly(styrenesuflonate) (PEDOT:PSS), Glycerol, Ethylene glycol, 1-ethyl-3-methylimidazolium ethyl sulfate >95\% (EMIM-ES), and Hydroxyethyl cellulose (HEC) were purchased from Sigma-Aldrich. $18.7 \mathrm{wt} \%$ Carboxymethylated Pulp cellulose in water was provided by RISE Bioeconomy.

Aluminium current collectors were provided by Dry Phase Patterning AB. Carbon microcircuit materials 7102 conductive paste ink was purchased from Dupont and the lacquer/insulating opaque white ultraswitch UVSW 170 ink was purchased from Marabu. A $500 \mu \mathrm{m}$ stencil frame was purchased from HPetch and screen frames were purchased from Coated Screens.

\section{Ink formulation}

The carbon and lacquer were used as received without any further modification.

The electrolyte was formulated by gelling the ionic liquid, EMIM-ES (21.2 wt\%) with HEC (6.2 wt\%) and DI water (72.5 wt \%). The EMIM-ES and DI water were mixed together using magnetic stirring and then placed on a hot plate at 100 degrees Celsius with the HEC slowly added. The mixture was allowed to thicken at 100 degrees Celsius for 1 hour. Once printed and dried the ionic gel becomes a sticky solid composed of only EMIM-ES (77.4 wt\%) and HEC (22.6 wt\%) since no evaporation of ionic liquid and cellulose is expected.

The power paper ink is based on PEDOT:Cellulose and was formulated similar to those mixed ionelectron conductor material systems reported previously. (18) However, dry PEDOT:PSS pellets (oragcon DRY) were substituted for the PEDOT:PSS aqueous suspension (Clevios PH 1000). Dimethyl sulfoxide was replaced by ethylene glycol as a safer alternative and pulp cellulose was substituted for the nanocellulose material. The PEDOT:Cellulose ink was composed of Orgacon DRY (1.9 wt\%), pulp 
cellulose (0.9 wt\%), glycerol (1.5wt\%), ethylene glycol (10.5 wt\%) and water (85.3 wt\%). The Orgacon DRY and pulp cellulose were mixed first before the ethylene glycol and glycerol were added followed by the water. The mixture was stirred magnetically for 24 hours before use. Following printing, most of the water and ethylene glycol evaporates leaving only the PEDOT:PSS, cellulose and glycerol, giving an approximative dry composition of 42.8, 21.9 and $34.3 \mathrm{wt} \%$, respectively.

\section{Printing processing}

The printing procedure was performed using an E2 EKRA screen-printing machine. Two layers of carbon (each layer was dried in an oven set at 80 degrees centigrade for 5 minutes) were screen printed onto the dry phase patterned aluminium electrodes followed by one layer of stencil printed PEDOT:Cellulose (dried in an oven set at 80 degrees centigrade for 20 minutes) to fabricate the electrodes. Two layers of lacquer ink were then screen printed (cured by UV light after each deposition) on the electrodes followed by an electrolyte layer stencil printed (dried in an oven set to 80 degrees centigrade for 20 minutes). Finally, the printed layers were manually laminated together to form functional supercapacitor devices. 16 half cells were fabricated simultaneously on the same substrate side by side.

\section{Supercapacitor Characterization}

Cyclic voltammetry, galvanostatic charge/discharge, coulombic efficiency and cyclability studies were all performed using a potentiostat (Ivium OctoStat200) and the Ivium Soft software.

Cyclic voltammetry was performed on the printed supercapacitors using scan rates of $50 \mathrm{mV} / \mathrm{S}, 100$ $\mathrm{mV} / \mathrm{s}$ and $500 \mathrm{mV} / \mathrm{s}$ from $0 \mathrm{~V}$ to $0.8 \mathrm{~V}$. Different voltage windows $(0.4,0.6,0.8,1.0,1.2$ and $1.4 \mathrm{~V})$ were then measured with a scan rate of $100 \mathrm{mV} / \mathrm{s}$.

Coulombic efficiency of the printed supercapacitor devices was calculated from the galvanostatic charge/discharge measurements with limit voltages set at $0.4,0.6,0.8,1.0,1.2$, and $1.4 \mathrm{~V}$ and was calculated using Eq. 1:

$$
\text { Coluombic efficiency }=\frac{\text { Discharge time }(s)}{\text { Charge time }(s)}
$$


Galvanostatic charge/discharge measurements were performed using current density (with respect to the mass of PEDOT on one electrode) values of $1 \mathrm{~A} / \mathrm{g}, 2 \mathrm{~A} / \mathrm{g}, 5 \mathrm{~A} / \mathrm{g}$ and $10 \mathrm{~A} / \mathrm{g}$ with a voltage limit of 0.8 $\mathrm{V}$ to ensure maximum stability ( $\mathrm{CV}$ measurements show potentially electrochemical side reactions above $0.8 \mathrm{~V}$ ). The capacitance and equivalent series resistance (ESR) were calculated from the discharge curves using Eq. 2 and Eq. 3, respectively.

$$
\begin{gathered}
\text { Capacitance }=\frac{\Delta t \times I}{V} \\
E S R=\frac{V_{d}}{2 \times I}
\end{gathered}
$$

Where $\Delta t$ is the discharge time, $\mathrm{I}$ is the current, $\mathrm{V}_{\mathrm{d}}$ is the voltage drop at the start of the discharge cycle and $\mathrm{V}$ is the voltage window excluding the voltage drop. Specific capacitance was calculated with respect of the mass of one PEDOT electrode and were corrected by a factor of two for literature comparison with 3 electrode systems. Specific energy and power were calculated using Eq. 4 and Eq. 5, respectively.

$$
\begin{array}{ll}
\text { Specific Energy }=\frac{1}{2 m} C V^{2} & \text { (Eq. 4) } \\
\text { Specific Power }=\frac{E}{\Delta t} & \text { (Eq. 5) }
\end{array}
$$

Where $\mathrm{m}$ is the mass of one PEDOT electrode (i.e. the mass of the electroactive material), $\mathrm{C}$ is the capacitance, $\mathrm{V}$ is the voltage, $\mathrm{E}$ is the specific energy and $\Delta \mathrm{t}$ is the discharge time.

Cyclability studies were performed by galvanostatic charge/discharge measurements on single cell supercapacitors to monitor degradation over time and cycles. These studies were carried out at a $2 \mathrm{~A} / \mathrm{g}$ current density $(10 \mathrm{~mA})$ for 10,000 cycles with a limit voltage of $0.8 \mathrm{~V}$.

On-demand manufactured supercapacitors were characterized by galvanostatic charge/discharge measurements and cyclic voltammetry to characterize current/capacitance and the voltage tolerance resulting from several supercapacitor cells connected in series. The supercapacitor circuit possessing 16 cells in parallel was charged/discharged at $0.1,0.25,0.5,1$ and $2 \mathrm{~A} / \mathrm{g}(8,20,40,80$ and $120 \mathrm{~mA})$ with a limit voltage of $0.8 \mathrm{~V}$ and cyclic voltammetry was performed within a voltage window of 0.8 and $1.6 \mathrm{~V}$ 
at a scan rate of $100 \mathrm{mV} / \mathrm{s}$. The supercapacitor circuits including 4 supercapacitors in series (each one composed of 4 individual parallel cells, each) were charged/discharged at $0.25,0.5$ and $1 \mathrm{~A} / \mathrm{g}(5,10$ and $20 \mathrm{~mA}$ ) with a limit voltage of $0.8 \mathrm{~V}$ and cyclic voltammetry was performed with a voltage window of $0.8,1.6,3.2,4$ and $4.8 \mathrm{~V}$ with a scan rate of $100 \mathrm{mV} / \mathrm{s}$. The supercapacitor circuits based on 16 individual supercapacitors in series were charged/discharged at $0.25,0.5$ and $1 \mathrm{~A} / \mathrm{g}(1.25,2.5$ and $5 \mathrm{~mA})$ with a limit voltage of $0.8 \mathrm{~V}$ and cyclic voltammetry was performed at voltage windows of 3.2, 6.4, 8.5 and $12.8 \mathrm{~V}$.

\section{Results and Discussion}

\section{Ink development}

The PEDOT:Cellulose ink developed retains the interesting properties observed in previous publications $(19,27)$ with the cellulose component allowing the electroactive composite to be robust, scalable and mechanically stable while in contact with an electrolyte. X-ray photon spectroscopy, atomic force microscopy and wide angle X-ray scattering have all been performed previously on similar PEDOT:PSS-cellulose composites to investigate the interesting properties of the material.(18) The ability of cellulose to form microscopic networks has been reported to be integral to this stability and robustness (Figure 1). Without the cellulose included in the formulation, it is impossible to print several PEDOT:PSS layers on top of each other, since cracking and delamination causes the electrode to fail. PEDOT:PSS has previously been used as the electrodes in symmetric supercapacitors, but only using thin $(\sim 10 \mu \mathrm{m})$ electrodes.(28) With the addition of the cellulose, the resulting "thick" $(\sim 100 \mu \mathrm{m})$ film electrode remains intact and robust, thus allowing the electrode to be included in a supercapacitor configuration. 
A)

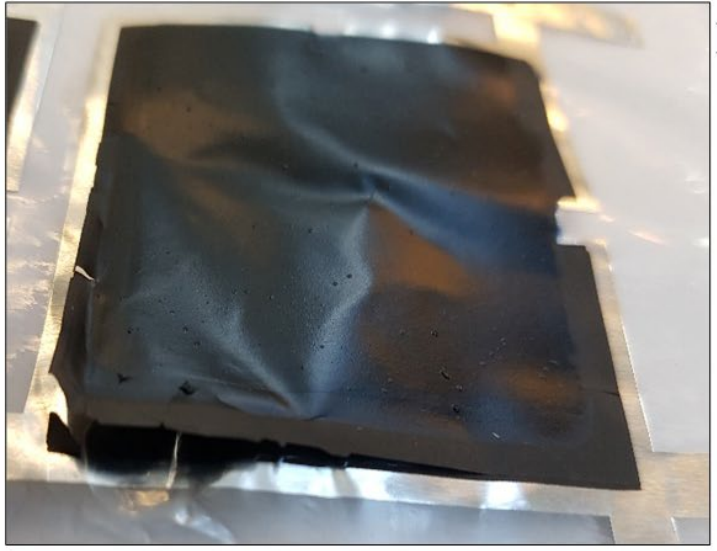

B)

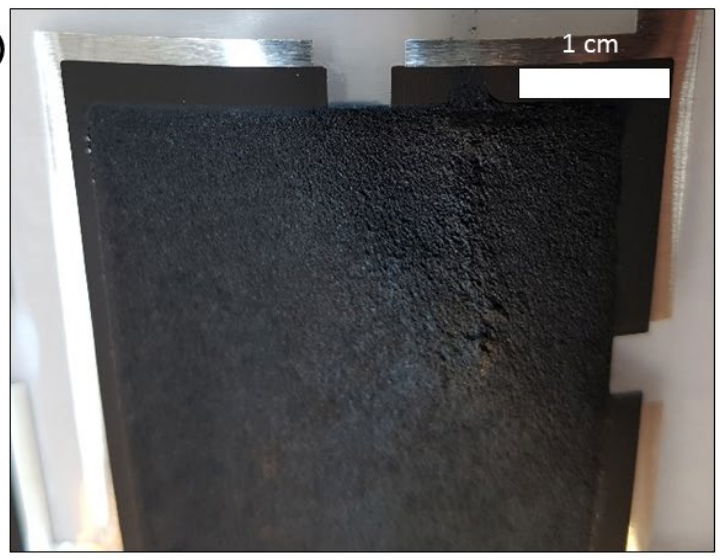

Figure 1. Photograph showing A) a PEDOT:PSS electrode without cellulose that results in cracking and delaminating due to internal stresses and lack of integrity and B) a PEDOT thick film with cellulose that shows no cracking or delamination due to the binding and integrity-promoting properties of cellulose. In both cases, 4 individual ink layers were stencil-printed and dried, on top of each other.

The optimization of the electrolyte included exploration and testing of various formulations. In particular, our focus was directed to explore and compare aqueous-based ( $\mathrm{LiCl})$ and ionic liquid-based electrolytes (EMIM-ES). Figure 2 presents an investigation of corrosion performed on supercapacitors based on different types of electrolytes during charge/discharge cycling. The aqueous-based electrolyte starts to corrode the aluminium collector already after $\sim 24$ hours despite including a carbon protective layer between the collector and the organic electrode material. At 48 hours these supercapacitors show severe degree of corrosion, as compared to the EMIM-ES-based supercapacitors, in addition to that they exhibit inflation due to internal gas production (Figure 2). In contrast, supercapacitors based on either neat EMIM-ES or gelled EMIM-ES electrolytes, do not corrode the aluminium electrodes or exhibit any inflation due to gas production. The gelled ionic liquid-based electrolyte was further developed for production, since the neat EMIM-ES electrolyte does not possess suitable rheologic properties to enable screen- or stencil printing processing. A gelled electrolyte is also advantageous since it supresses electrolyte leakage, which is a common device-failure mechanism. The rheology properties of the two ionic liquid-based electrolytes are given in the supporting information (Figure SI1). 


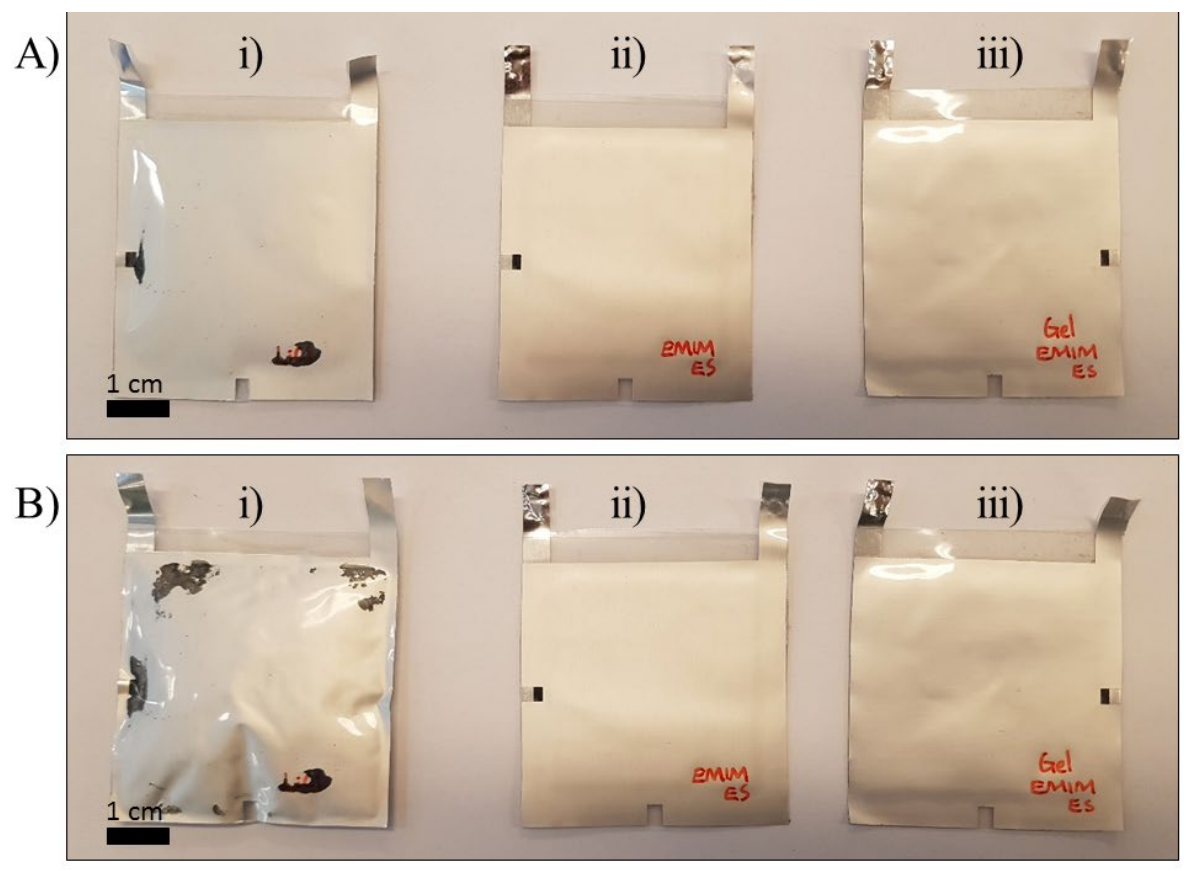

Figure 2. Corrosion investigation of electrolytes after A) 24 hours and B) 48 hours. i) $\mathrm{LiCl}$ aqueous based electrolyte, ii) Neat EMIM-ES and iii) EMIM-ES gelled with HEC. The corrosion appears as darkened areas on the aluminium surface.

\section{Printing processing}

Design of the printed supercapacitor structures were performed targeting printability, customization and optimal supercapacitor performance. Our chosen design allows for a layer-by-layer deposition approach, to achieve scalable electrodes, followed by a lamination step of two electrodes sheets to form the resulting supercapacitor. Thus, the process can be upscaled into a fully printed production process, thus enabling low costs and swift throughput.

Printing manufacturing allows for reproducibility and scalability of electronic components and energy devices. Screen printing machines such as the EKRA, shown in Figure 3 B) permits single sheets of PET (and other substrate materials) to be coated with inks for devices, based on several stacked layers, such as supercapacitors. Many sheets can be coated in a short time, thanks to semi-automatic operation, with manual labour only needed for handling of sheets and for replenishment of the ink. The inclusion of alignment marks in the screen design (Figure 3 A) and supporting information) allows for the manufacturing of multilayered devices at registration of approximately $0.2 \mathrm{~mm}$. The EKRA screen 
printer is designed suitable for prototype and pilot screen printing of patterns and devices, before transferring the production protocol to large-scale manufacturing.

The printing procedure was optimised to manufacture supercapacitors that do not exhibit any electrical shorts, between the two laminated electrodes, and that display an overall high performance in electrical characteristics. The current collectors were generated using dry phase patterning, which is a commercialized manufacturing procedure which mills laminated aluminium foils into desired patterns and structures. Two sequential layers of carbon was deposited on top of each aluminium collector pattern, in order to protect the collector from corrosion. The second deposited layer of carbon fills any potential pin holes generated in the first printing steps, thus providing a suitable protection of the aluminium collector. In the next step, the PEDOT:PSS-Cellulose electrode material is deposited onto the collector via stencil printing, and the amount of material deposited can be altered depending on the application and specifications. For thin supercapacitors, only one layer is printed and for applications requiring higher capacitance levels several layers are deposited. For the results published here, we have chosen to report data for supercapacitors based on one layer only. Two lacquer protection layers were also screen printed onto the remaining exposed aluminium surface to ensure that no electrical shortening occur during operation of the supercapacitors (Figure $3 \mathrm{C}$ ).

The EMIM-ES gel electrolyte was initially screen printed by applying 3 sequential layers on top of each other. However, electrical shorts were still encountered frequently. We speculate that electrolyte is partially soaked into the PEDOT:Cellulose electrode layer allowing the two opposing PEDOT:Cellulose layers to physically come in contact with each other. Therefore, a thicker electrolyte layer is necessary and stencil printing provides a solution. An alternative to thicker electrolytes is to consider the inclusion of a separator. However, our tests on combining a separator with screen printed gels did result in poor performance. The addition of a separator would also increase the complexity in the manufacturing process since a "pick-and-place" step is introduced. The physical dimension of the resulting supercapacitor also increases as the separator adds volume and mass to the devices structure. A schematic visualization of the layers along with photographs of the printed layers are given in Figure 3 C) and D), respectively. 


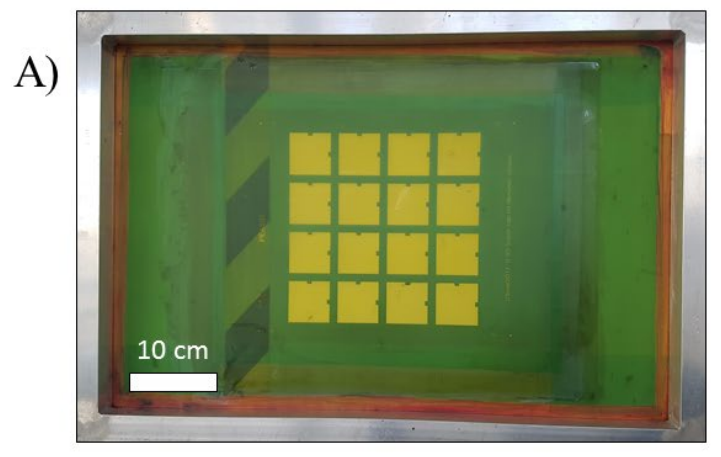

C)

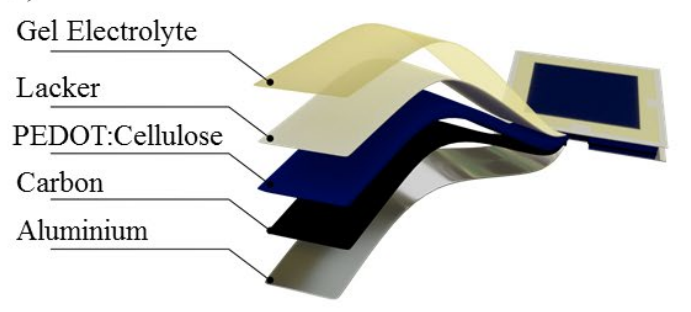

B)

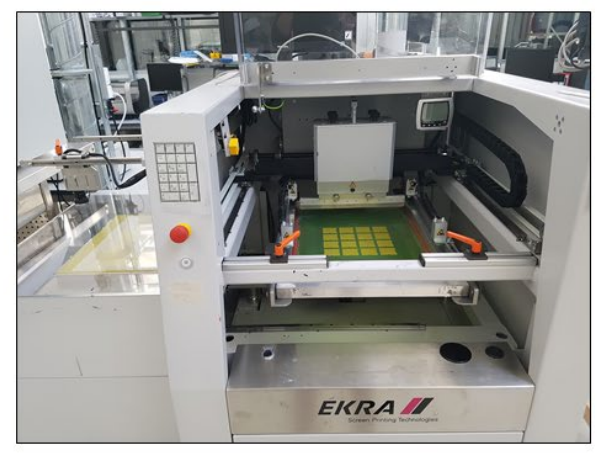

D)

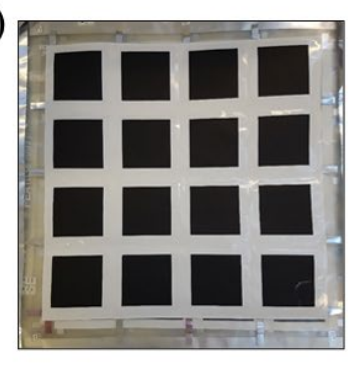

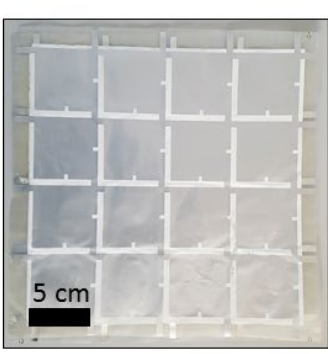

Figure 3. Photographs of A) Screen printing frame with supercapacitor design (all screen printing and stencil designs can be found in supporting information) and B) the EKRA, screen printing machine, C) schematic showing the screen and stencil printed layers (one side of the symmetrical energy storage device) D) One complete side of 16 printed supercapacitors before lamination and after lamination. Photographs of every component deposited can be seen in the supporting information.

\section{Supercapacitor Performance}

Columbic efficiency was calculated from the galvanostatic charge/discharge curves, data are given in the supporting information (Figure SI6). The efficiency remained above $97 \%$ even for high voltages of $1.4 \mathrm{~V}$. However, from cyclic voltammetry measurements performed on individual supercapacitors, deviations from the ideal capacitive (box-like) behaviour are observed for voltages above $0.8 \mathrm{~V}$ (Figure $4 \mathrm{~A}$ ). This could indicate the onset of electrochemical overoxidation of PEDOT. Therefore, all further electrical characterizations were carried out for voltages limited to below $0.8 \mathrm{~V}$ per supercapacitor cell. The scan rate was varied in the cyclic voltammetry measurements but only little change with respect to capacitance values was shown (Figure $4 \mathrm{~B}$ ).

The galvanostatic charge/discharge curves are given in Figure $4 \mathrm{C}$ for an individual printed supercapacitor at various current density levels (ranging from 1 to $10 \mathrm{~A} / \mathrm{g}$ ). With an active electrode 
mass of approximately $5 \mathrm{mg}$ per PEDOT:Cellulose (calculated by mass of ink deposited) electrode the capacitance and specific capacitance agrees well with previously reported data (23), and here gives us values ranging from 218-230 $\mathrm{mF}$ and $87-92 \mathrm{~F} / \mathrm{g}$, respectively (Figure $4 \mathrm{D}$ ). Since the specific capacitance is comparable to that of thin film PEDOT:PSS electrodes (22), the entire bulk of the electrode (PEDOT:PSS) is taking part in the charge storage mechanism. The lifetime of the supercapacitors performs well over many cycles. The devices underwent 10,000 charge/discharge cycles and only very little degradation was observed after all cycles (Figure $4 \mathrm{E}$ ). The initial dip in capacitance is rationalized to electrochemical side reactions, likely caused by absorbed water and/or oxygen in the electrodes and electrolyte since the devices were manufactured in ambient atmosphere. Figure SI7 shows a sharp increase in the inputted charge (C) during the galvanostatic cycling which then fall back to the initial value. This behaviour correlates to the decrease in capacitance followed by recovery as the reactants are being depleted in the devices. The specific energy and power performance of the all-printed supercapacitors were then also characterized (Figure 4 F). The yellow star, in Figure 4 F, compares an individual printed supercapacitor to the performance of other energy storage device technologies. 

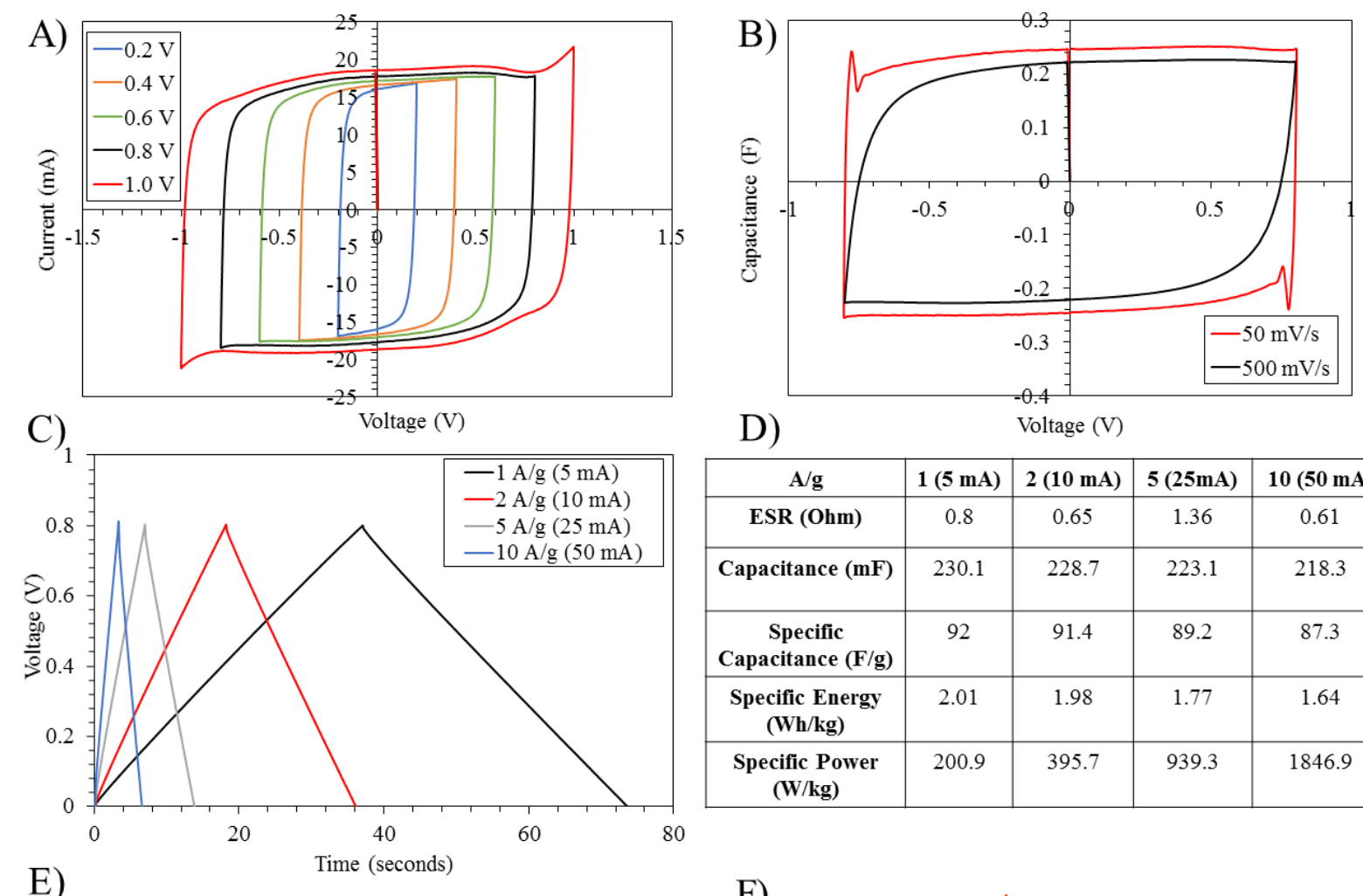

\begin{tabular}{|c|c|c|c|c|}
\hline D) & \multicolumn{5}{|c|}{ Voltage (V) } \\
\hline $\mathbf{A} / \mathbf{g}$ & $\mathbf{1}(\mathbf{5} \mathbf{~ m A})$ & $\mathbf{2}(\mathbf{1 0} \mathbf{~ m A})$ & $\mathbf{5}(\mathbf{2 5 m A})$ & $\mathbf{1 0} \mathbf{( 5 0 ~ \mathbf { ~ A } )}$ \\
\hline ESR (Ohm) & 0.8 & 0.65 & 1.36 & 0.61 \\
\hline Capacitance (mF) & 230.1 & 228.7 & 223.1 & 218.3 \\
\hline $\begin{array}{c}\text { Specific } \\
\text { Capacitance (F/g) }\end{array}$ & 92 & 91.4 & 89.2 & 87.3 \\
$\begin{array}{c}\text { Specific Energy } \\
\text { (Wh/kg) }\end{array}$ & 2.01 & 1.98 & 1.77 & 1.64 \\
\hline $\begin{array}{c}\text { Specific Power } \\
\text { (W/kg) }\end{array}$ & 200.9 & 395.7 & 939.3 & 1846.9 \\
\hline
\end{tabular}

E)

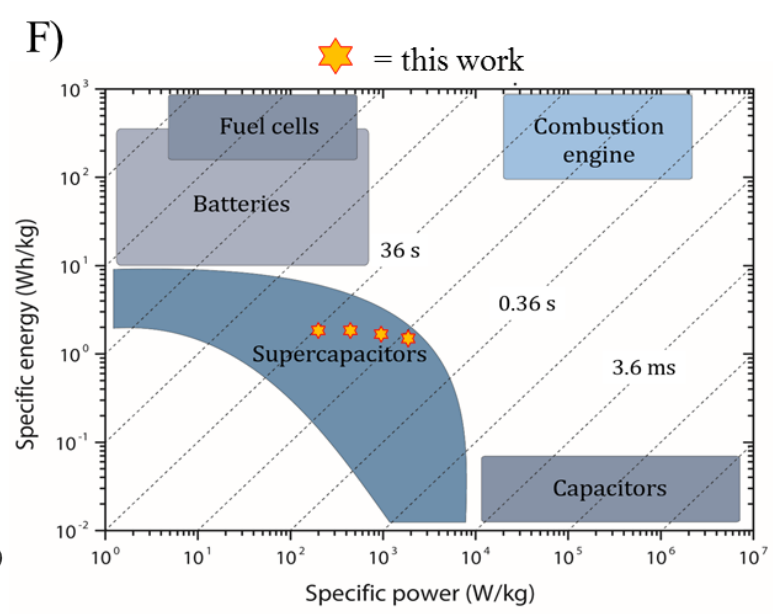

Figure 4. Supercapacitor performance characteristics of the all printed supercapacitors. A) CV curves at different voltage windows (Scan rate $=100 \mathrm{mV} / \mathrm{s}$ ) showing the capacitive nature and the beginning of electrochemical degradation, B) CV curves at different scan rates, C) galvanostatic charge/discharge curves at different current levels for capacitance calculations. D) Table showing the supercapacitor properties from the charge/discharge curves, E) Cyclability of the supercapacitors over 10,000 cycles and F) Ragone plot showing where this work relates to the conventional energy storage devices.

\section{Supercapacitors on demand}


The conceptual idea of the supercapacitors-on-demand is illustrated in Figure 5 A-C. A full set of 16 supercapacitors are manufactured in a $4 \times 4$ device matrix configuration, according to the procedure outlined above. In its pristine configuration all 16 electrodes on each side are connected to each other, thus making all the 16 supercapacitors connected in parallel (Figure $5 \mathrm{~A}$ ). A punching tool was used to terminate individual connections in between lateral electrodes, thus enabling us to increase the operational voltage and to reduce the capacitance, on demand, such as configuring 4 or 16 supercapacitors in series (Figure $5 \mathrm{~B}$ and C). All three different configurations are shown in Figure 5 AC. Schematics showing both sides of the supercapacitors and how they are cut for different configurations can be found in supporting information along with the characterization of each device.
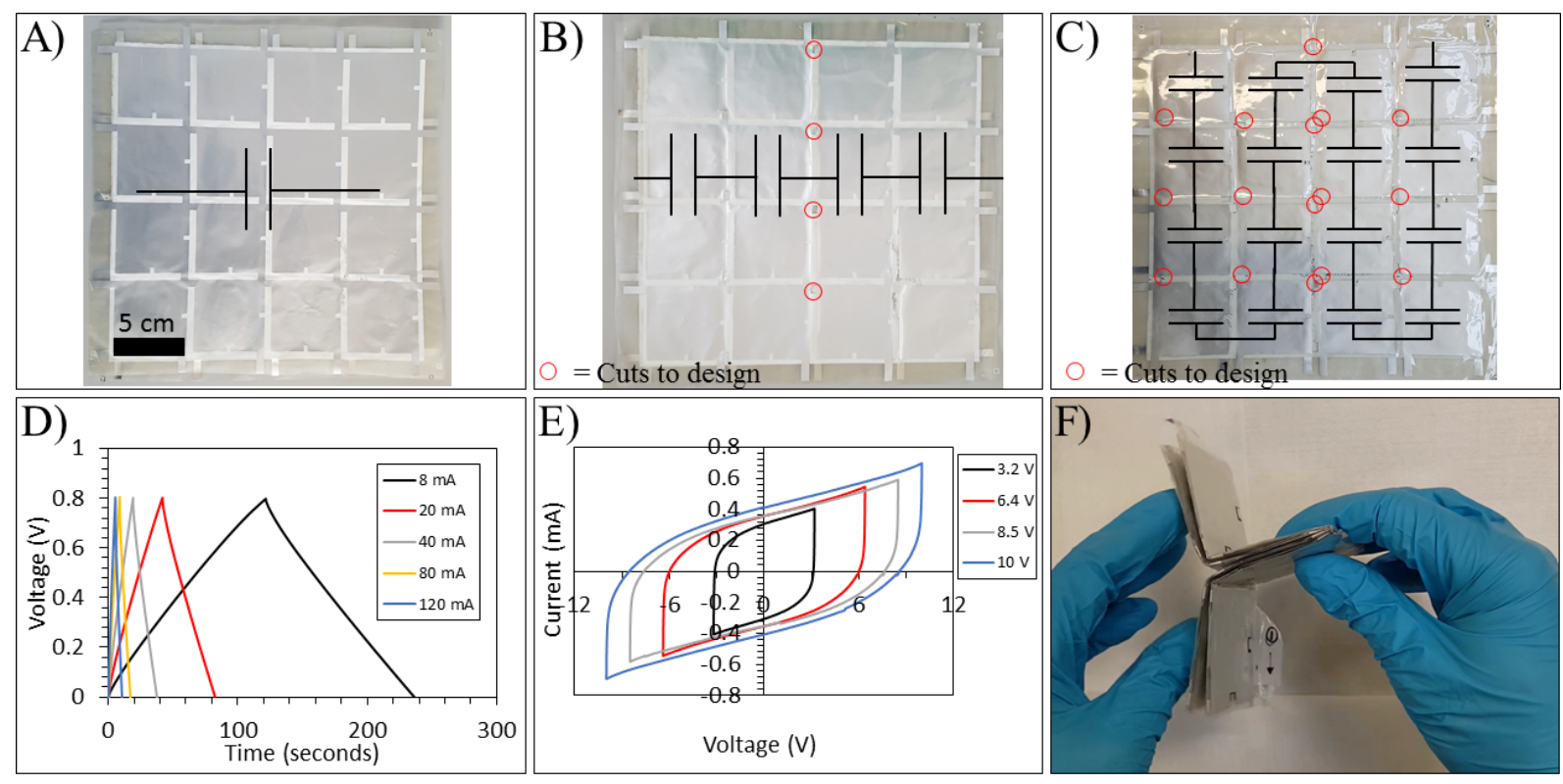

Figure 5. Example of the supercapacitor on demand concept as A) 16 cells in parallel, B) 4 cells in series (each composed of 4 cells in parallel) and C) 16 cells in series for adaption for various applications and voltage requirements. D) Galvanostatic charge/discharge curves of the first configuration allowing larger currents to be used E) Cyclic voltammetry chromatographs using the 16 individual supercapacitors in series to increase the voltage and F) Photograph highlighting the foldable and conformable properties of the all printed supercapacitors. Further characterization can be found in the supporting information. 
The resulting supercapacitor without any cuts introduced (Figure $5 \mathrm{~A}$ ) represents a 16 times upscaled version of the individual cell characterized earlier and presented in Figure 4. This device allows higher currents for charging and discharging due to the increased amount of active material (Figure 5 D). When specific cut have been introduced, using the punching tool, supercapacitors are configured in series (Figure $5 \mathrm{~B}$ and $\mathrm{C}$ ) the safe voltage of operation (before electrochemical reactions reduce cyclability) increases. For the configuration of 16 supercapacitors in series, the operational voltage window can be increased up to $10 \mathrm{~V}$, see Figure 5 E. Cyclic voltammetry characterizations of each device can be found in the supporting information (Figure SI9 and SI10). Since the $4 \times 4$ supercapacitor matrix is produced on flexible foils, the resulting system remains flexible and foldable for convenient packaging. By simply folding the matrix substrate 4 times, a convenient package of post-productionconfigured supercapacitors are at hand (Figure $5 \mathrm{~F}$ ). Figure SI1 1 shows the results of a printed supercapacitor before, during and after folding without any significant change to the performance of the device. Thus, all-printed, flexible and configurable organic supercapacitors have been developed, characterized and developed.

\section{Conclusion}

In this report we present energy storage devices based on the power paper ink; an ink system based on PEDOT:Cellulose which serves as the electrode material in all-printed and flexible supercapacitors that are possible to customize depending on the specifications and need of the targeted applications. The supercapacitors are based on carbon-aluminium collectors, on flexible foils, onto which one or several layers of the power paper ink is deposited using stencil printing. Then, a layer of thick gelled ionic liquid is deposited, to serve as the electrolyte, followed by lacquer deposition and finally lamination encapsulation. The supercapacitors can be patterned to form many individual but interconnected supercapacitors arranged into a matrix. To supply the market with an energy storage technology on a highly differentiated market, it is important that the technology enables flexible design so that a wide range of specifications can be reached with relatively small changes in the production protocol. The volume of the electrode material, which dictate the capacitance value of the supercapacitor, can be tailormade by just adding or excluding stencil printing steps of the power paper ink. By using a punching 
tool, the possibility arises to specify the operational characteristics of the supercapacitor even after completed production. Individual printed supercapacitors were characterized showing good performance in terms of specific capacitance $(\approx 90 \mathrm{~F} / \mathrm{g})$, cycle life $(>10,000$ cycles $)$ and specific energy and power. The supercapacitor-on-demand concept was demonstrated using three different configurations allowing us to dictate the resulting current and operational voltage window, up to 120 $\mathrm{mA}$ and $10 \mathrm{~V}$, respectively.

\section{Acknowledgements}

The authors would like to thank DPP AB for supplying the electrode materials and RISE Bioeconomy for supplying the cellulose materials. This work was financially supported by the Swedish Foundation for Strategic Research (GMT14-0058).

\section{References}

1. Song Z, Zhou H. Towards sustainable and versatile energy storage devices: an overview of organic electrode materials. Energy \& Environmental Science. 2013;6(8):2280-301.

2. Bandodkar AJ, You J-M, Kim N-H, Gu Y, Kumar R, Mohan AMV, et al. Soft, stretchable, high power density electronic skin-based biofuel cells for scavenging energy from human sweat. Energy \& Environmental Science. 2017;10(7):1581-9.

3. Sun H, Xie S, Li Y, Jiang Y, Sun X, Wang B, et al. Large-Area Supercapacitor Textiles with Novel Hierarchical Conducting Structures. Advanced Materials. 2016;28(38):8431-8.

4. Liu Y-H, Xu J-L, Gao X, Sun Y-L, Lv J-J, Shen S, et al. Freestanding transparent metallic network based ultrathin, foldable and designable supercapacitors. Energy \& Environmental Science. 2017;10(12):2534-43.

5. Choi K-H, Ahn DB, Lee S-Y. Current Status and Challenges in Printed Batteries: Toward Form Factor-Free, Monolithic Integrated Power Sources. ACS Energy Letters. 2018;3(1):220-36.

6. Hoeng F, Denneulin A, Bras J. Use of nanocellulose in printed electronics: a review. Nanoscale. 2016;8(27):13131-54.

7. Tuukkanen S, Lehtimäki S, Jahangir F, Eskelinen A, Lupo D, Franssila S, editors. Printable and disposable supercapacitor from nanocellulose and carbon nanotubes. Proceedings of the 5 th Electronics System-integration Technology Conference (ESTC); 2014 16-18 Sept. 2014.

8. Sani N, Linderhed U, Sandberg M. Monolithically integrated electrochemical energy storage modules. Journal of Energy Storage. 2018;16:139-44.

9. Pereira RBaICaDGaEFaRMaL. Printable cellulose-based electroconductive composites for sensing elements in paper electronics. Flexible and Printed Electronics. 2017;2(1):014006.

10. Cobb CL, Ho CC. Additive Manufacturing: Rethinking Battery Design. The Electrochemical Society Interface. 2016;25(1):75-8.

11. Kumar R, Shin J, Yin L, You J-M, Meng YS, Wang J. All-Printed, Stretchable Zn-Ag2O Rechargeable Battery via Hyperelastic Binder for Self-Powering Wearable Electronics. Advanced Energy Materials. 2016;7(8):1602096.

12. Gaikwad AM, Steingart DA, Nga Ng T, Schwartz DE, Whiting GL. A flexible high potential printed battery for powering printed electronics. Applied Physics Letters. 2013;102(23):233302. 
13. Xiong Z, Liao C, Han W, Wang X. Mechanically Tough Large-Area Hierarchical Porous Graphene Films for High-Performance Flexible Supercapacitor Applications. Advanced Materials. 2015;27(30):4469-75.

14. Chen B, Jiang Y, Tang X, Pan Y, Hu S. Fully Packaged Carbon Nanotube Supercapacitors by Direct Ink Writing on Flexible Substrates. ACS Applied Materials \& Interfaces. 2017;9(34):28433-40.

15. Tang Z, Zhang G, Zhang H, Wang L, Shi H, Wei D, et al. MOF-derived N-doped carbon bubbles on carbon tube arrays for flexible high-rate supercapacitors. Energy Storage Materials. 2018;10:75-

84.

16. Sollami Delekta S, Smith AD, Li J, Östling M. Inkjet printed highly transparent and flexible graphene micro-supercapacitors. Nanoscale. 2017;9(21):6998-7005.

17. Brooke R, Fabretto M, Krasowska M, Talemi P, Pering S, Murphy PJ, et al. Organic energy devices from ionic liquids and conducting polymers. Journal of Materials Chemistry $C$. 2016;4(7):1550-6.

18. Malti A, Edberg J, Granberg H, Khan ZU, Andreasen JW, Liu X, et al. An Organic Mixed IonElectron Conductor for Power Electronics. Advanced Science. 2015;3(2):1500305.

19. Edberg J, Inganäs O, Engquist I, Berggren M. Boosting the capacity of all-organic paper supercapacitors using wood derivatives. Journal of Materials Chemistry A. 2018;6(1):145-52.

20. Andersson Ersman P, Kawahara J. Roll to roll printable technology platform consisting of electrolyte-based components. Printed Electronics at Acreo, published in LOPE-C 2011 Conference Proceedings; 20112011.

21. Andersson P, Forchheimer R, Tehrani P, Berggren M. Printable All-Organic Electrochromic Active-Matrix Displays. Advanced Functional Materials. 2007;17(16):3074-82.

22. Ajjan FN, Casado N, Rębiś T, Elfwing A, Solin N, Mecerreyes D, et al. High performance PEDOT/lignin biopolymer composites for electrochemical supercapacitors. Journal of Materials Chemistry A. 2016;4(5):1838-47.

23. Wang Z, Tammela P, Huo J, Zhang P, Strømme M, Nyholm L. Solution-processed poly(3,4ethylenedioxythiophene) nanocomposite paper electrodes for high-capacitance flexible supercapacitors. Journal of Materials Chemistry A. 2016;4(5):1714-22.

24. Liu I, Lu Q, Yang S, Guo J, Tian Q, Yao W, et al. All-Printed Solid-State Microsupercapacitors Derived from Self-Template Synthesis of Ag@PPy Nanocomposites2017. 1700206 p.

25. Kawahara J, Ersman P, Wang X, Gustafsson G, Granberg H, Berggren M. Reconfigurable sticker label electronics manufactured from nanofibrillated cellulose-based self-adhesive organic electronic materials2013.

26. Malti A, Brooke R, Liu X, Zhao D, Andersson Ersman P, Fahlman M, et al. Freestanding electrochromic paper. Journal of Materials Chemistry C. 2016;4(41):9680-6.

27. Edberg J, Malti A, Granberg H, M Hamedi M, Crispin X, Engquist I, et al. Electrochemical circuits from 'cut and stick' PEDOT:PSS-nanocellulose composite2017. $045010 \mathrm{p}$.

28. Liu Y, Weng B, Razal J, Xu Q, Zhao C, Hou Y, et al. High-Performance Flexible All-Solid-State Supercapacitor from Large Free-Standing Graphene-PEDOT/PSS Films2015. 17045 p. 